\title{
Analyzing Taiwan IC Assembly Industry by Grey-Markov Forecasting Model
}

\author{
Lei-Chuan Lin and Shan-Yau Wu \\ Department of Industrial Engineering and Management, National Kaohsiung University of Applied Sciences, \\ Kaohsiung 80778, Taiwan
}

Correspondence should be addressed to Lei-Chuan Lin; leichuan@mail.mirdc.org.tw

Received 14 September 2013; Revised 19 October 2013; Accepted 20 October 2013

Academic Editor: Teen-Hang Meen

Copyright (C) 2013 L.-C. Lin and S.-Y. Wu. This is an open access article distributed under the Creative Commons Attribution License, which permits unrestricted use, distribution, and reproduction in any medium, provided the original work is properly cited.

\begin{abstract}
This study utilizes the black swan theorem to discuss how to face the lack of historical data and outliers. They may cause huge influences which make it impossible for people to predict the economy from their knowledge or experiences. Meanwhile, they cause the general dilemma of which prediction tool to be used which is also considered in this study. For the reason above, this study uses 2009 Q1 to 2010 Q4 quarterly revenue trend of Taiwan's semiconductor packaging and testing industry under the global financial turmoil as basis and the grey prediction method to deal with nonlinear problems and small data. Under the lack of information and economic drastic changes, this study applies Markov model to predict the industry revenues of GM(1,1) and DGM $(1,1)$ results. The results show that the accuracy of 2010 Q1-Q3 is $88.37 \%, 90.27 \%$, sand $91.13 \%$, respectively. Besides, they are better than the results of $\operatorname{GM}(1,1)$ and DGM $(1,1)$ which are $86.51 \%, 77.35 \%, 75.46 \%$ and $73.77 \%, 74.25 \%, 59.72 \%$. The results show that the prediction ability of the grey prediction with Markov model is better than traditional GM(1,1) and DGM(1,1) sfacing the changes of financial crisis. The results also prove that the grey-Markov chain prediction can be the perfect criterion for decision-makers judgment even when the environment has undergone drastic changes which bring the impact of unpredictable conditions.
\end{abstract}

\section{Introduction}

Financial turmoil has huge influences on varieties of industries, like the black swan theorem which produces a great deviation and causes adverse reactions [1]. In Taiwan, semiconductor production capacity accounts for more than half of the global semiconductor manufacturing market, and the IC packaging and testing accounts for the global foundry capacity of over $60 \%$. The speed and environment of new products replacing older market create the competitiveness of related industries in Taiwan. The black swan theorem will produce outliers. This study found that the way to remove the deviation would be more difficult to predict regression or other methods of forecasting future trends accurately.

The grey method proposed by professor Long can provide very good prediction under short-time historical data [2]. Among the method, $\mathrm{GM}(1,1)$ provides suitable reference tools to decision-makers in the changing and competitive environment. In the previous researches, grey theory applied to the economic, financial, engineering, and other fields reached ideal results [3-5]. For example, Ken and Li [6] used a different grey prediction model which included traditional $\operatorname{GM}(1,1), \operatorname{RGM}(1,1)$, and Tan's GM(1,1), and he used chained convenience stores sales patterns as backgrounds for model selection. Hung et al. [7] also used GM(1,1) to predict under incomplete information. Compared with two prediction models of Egli and Walfisch-bertoni [8], it had proved that the $\mathrm{GM}(1,1)$ rolling model produced the lowest error. Lin and Yang [9] used GM(1,1) to predict Taiwan semiconductor optoelectronics industry, and the average residual is less than $10 \%$, which proves the high accuracy of grey prediction to semiconductor industry. But the financial crisis causes excessive fluctuations in random sequence and it easily leads to the condition of inadequate accuracy. If it is applied to the Taiwan semiconductor packaging and testing industry revenue data, it will cause the lack of precision because of deficiencies historical data message with random fluctuations, which also increases the difficulty in using general prediction tools in this paper. 
Markov (Markov-chain) is generally used in the forecasting model on a random time series, and it is a dynamic system which is based on the transition between the states and all the random factors to reflect the impact [7]. Although it is very suitable for handling random variation problems, it still needs enough raw data to determine the probability of state transitions. Hence, this study uses Markov theory to improve $\operatorname{GM}(1,1)$ and reduce the variation when historical data increase and financial crisis happens. By evaluating accuracy, the prediction effect of Markov model can be improved [3].

\section{Grey System Theory}

Yin [10] has investigated during the time span of 1996 to 2011, the earliest year when the database was available to the best possible full text availability to date. The paper points out that there were 482 publications that met the selection criteria of "grey relational analysis" or "gray relational analysis" topic search. It is seen that the grey system theory is one of the most popular forecasting methods. Ken and Li [6] applied grey system as a module to help predicting sales revenue of convenience chain stores in Taiwan. They do get more accuracy on their prediction. Also, Huang et al. [11] predict the silkworm cocoon yield in china base on the grey-Markov forecasting model. The findings show that grey-Markov has more accuracy than other forecasting model. Meanwhile, Hsu and Chen [12] compared various statistical methods based on the suitability of short-term forecasting. They got similar findings.

\section{Methodology}

3.1. GM(1,1) and DGM(1,1) Grey Forecasting Model. GM(1,1) is a type of prediction model, the analysis steps are listed below.

Step 1. The original sequence is defined as

$$
X^{(0)}=\left(x_{1}^{(0)}, x_{2}^{(0)}, \ldots, x_{n}^{(0)}\right) .
$$

Step 2. Use 1-AGO to decrease the randomness of sequence and define the 1-AGO as

$$
X^{(1)}=\left(x_{1}^{(1)}, x_{2}^{(1)}, \ldots, x_{n}^{(1)}\right),
$$

where $x_{k}^{(1)}=\sum_{i=1}^{k} x_{i}^{(0)}, k=1,2, \ldots, n$.

Step 3. Establish a first-order differential equation

$$
\frac{d X^{(1)}}{d t}+a X^{(1)}=u
$$

Step 4. From (3), we can get

$$
\begin{aligned}
\widehat{x}^{(1)}(k+1) & =\left(x^{(0)}(1)-\frac{u}{a}\right) e^{-a k}+\frac{u}{a}, \quad k \geq 0, \\
\widehat{a} & =\left[\begin{array}{l}
a \\
u
\end{array}\right]=\left(B^{T} B\right)^{-1} B^{T} Y,
\end{aligned}
$$

where

$$
\begin{gathered}
B=\left[\begin{array}{cc}
-\frac{1}{2\left(x_{1}^{(0)}+x_{2}^{(0)}\right)} & 1 \\
-\frac{1}{2\left(x_{2}^{(0)}+x_{3}^{(0)}\right)} & 1 \\
\vdots & \vdots \\
\frac{-1}{2\left(x_{n-1}^{(0)}+x_{n}^{(0)}\right)} & 1
\end{array}\right]=\left[\begin{array}{ccc}
-z^{(1)}(2) & 1 \\
-z^{(1)}(3) & 1 \\
\vdots & \vdots \\
-z^{(1)}(n) & 1
\end{array}\right], \\
Y=\left[\begin{array}{c}
x^{(0)}(2) \\
x^{(0)}(3) \\
\vdots \\
x^{(0)}(n)
\end{array}\right] .
\end{gathered}
$$

Step 5. Use 1-AGO to get the real prediction data

$$
\widehat{x}(k+1)=\widehat{x}^{(1)}(k+1)-\widehat{x}^{(1)}(k) .
$$

Step 6. From Step 5, a trend curve equation can be formed

$$
\widehat{Y}(k)=\widehat{x}^{(0)}(k+1)=g e^{-a k},
$$

where $g=\left(x^{(0)}(1)-(b / a)-e^{a} x_{(1)}^{(0)}+e^{a}(b / a)\right)$.

$\operatorname{DGM}(1,1)$ discrete grey forecasting model is the dynamic cycle rolling model; it is from original sequence by $\operatorname{GM}(1,1)$. Use this result of $\operatorname{DGM}(1,1)$ model to become the first stage of forecasting value that is needed. Comparative forecasting value and actual value are examined in order to get error value and accuracy.

\subsection{Partition of States by Markov-Chain Forecasting Model.} The values of $X^{(0)}(k+1)$ are distributed in the region of the trend curve $\widehat{Y}(k)$ that may be divided into a convenient number of contiguous intervals. When $X^{(0)}(k+1)$ falls in interval $i$, one of $S$ such intervals, it may be regarded as corresponding to a state $E_{i}$, and $E_{i}$ can be signified as follows:

$$
E_{i}=\left[E_{1 i}, E_{2 i}\right] \text {, }
$$

where $i=1,2, \ldots, S$ and $S$ is the amount of states

$$
\begin{aligned}
& E_{1 i}=\widehat{Y}(k)+A_{i}, \\
& E_{2 i}=\widehat{Y}(k)+B_{i},
\end{aligned}
$$

where $\widehat{Y}(k)$ is time function, $E_{i}$ is in the $i$ state, $E_{1 i}$ and $E_{2 i}$ are tine varying, and $A_{i}$ and $B_{i}$ are fixed value, depending on the original data.

3.3. Calculation of State Transfer Probability. In Markovchain series, the changing probability form $E_{i}$ to $E_{j}$ can be presented as follows:

$$
P_{i j}(m)=\frac{M_{i j}(m)}{M_{i}} \quad(i, j=1,2, \ldots, S),
$$


where $P_{i j}(m)$ is the transition probability of state $E_{j}$ transferred from state $E_{i}$ for $m$ steps (in this paper, 1 step stands for 1 quarter), $m$ is the number of transition steps each time, $M_{i j}(m)$ is the number of original data of state $E_{j}$ transferred from state $E_{i}$ for $\mathrm{m}$ steps, and $M_{i}$ is the number of original data points in state $E_{i}$.

The state transition probability matrix $R(m)$ is as follows:

$$
\begin{aligned}
& R(m)=\left[\begin{array}{llll}
p_{11}(m) & p_{12}(m) & \cdots & p_{1 j}(m) \\
p_{21}(m) & p_{22}(m) & \cdots & p_{2 j}(m) \\
& & \cdots & \\
p_{j 1}(m) & p_{j 2}(m) & \cdots & p_{i j}(m)
\end{array}\right] \\
& (i, j=1,2, \ldots, S) .
\end{aligned}
$$

The state transition probability $P_{i j}(m)$ reflects the transition rules of a system. The state transition probability matrix $R(m)$ describes the probability of transition from state $i$ to $j$. Generally, it is necessary to observe the one-step transition matrix $R(1)$. Supposing the object to be forecasted is in state $E_{Q}(1 \leq Q \leq S)$, row $Q$ in matrix $R(1)$ should be considered. If $\max P_{\mathrm{Qj}}(1)=P_{\mathrm{QL}}(1)(j=1,2, \ldots, S ; 1 \leq \mathrm{Q} \leq S)$, then what will most probably happen in the system at the next moment is the transition from state $E_{Q}$ to state $E_{L}$. It is difficult to determine the future transition of the state; if two or more transition probabilities in the row $Q$ of matrix $R(1)$ are the same, the transition probability matrix of two-step transition matrix $R(2)$ or multistep transition matrix $R(m)$, where $m \geq 3$, should be considered [4].

3.4. Calculate the Forecasting Data. After the determination of the future state transition of a system, that is, the determination of grey elements $E_{1 i}, E_{2 i}$, the changing interval of the forecast value is between $E_{1 i}$ and $E_{2 i}$. The most probable forecast value, $\widehat{Y}(k+1)$, is considered to be the middle value of the determined state interval; that is,

$$
\widehat{Y}(k+1)=\frac{1}{2\left(E_{1 i}+E_{2 i}\right)}=\widehat{Y}(k)+\frac{1}{2\left(A_{i}+B_{i}\right)} .
$$

3.5. Accuracy Inspection Analysis of Forecasting Ability. Numerous methods exist for judging forecasting model accuracy, and no single recognized inspection method exists for forecasting ability. MAPE is often used to measure forecasting accuracy. Smaller MAPE value indicates better forecasting ability

$$
\begin{aligned}
\text { MAPE } & =\frac{1}{n} \sum \frac{\mid \text { Actual }- \text { Forecast } \mid}{\text { Actual }} \times 100, \\
& n=\text { Forecasting number of step. }
\end{aligned}
$$

Evaluation of MAPE forecasting ability is divided as forecasting ability is evaluated as follows:

(i) $<10$ excellent forecasting ability,

(ii) 10 20 good forecasting ability,

(iii) 20 50 reasonable forecasting ability,

(iv) $>50$ poor forecasting.
TABLE 1: The IC assembly industry revenue of Taiwan from 2007 Q1 to $2010 \mathrm{Q} 3$.

\begin{tabular}{lcc}
\hline Number & Year & Amount \\
\hline 1 & 2007 Q1 & $39,317,687$ \\
2 & 2007 Q2 & $42,373,477$ \\
3 & 2007 Q3 & $49,468,262$ \\
4 & 2007 Q4 & $50,574,715$ \\
5 & 2008 Q1 & $43,602,058$ \\
6 & 2008 Q2 & $46,390,298$ \\
7 & 2008 Q3 & $48,554,800$ \\
8 & 2008 Q4 & $36,766,363$ \\
9 & 2009 Q1 & $28,271,646$ \\
10 & 2009 Q2 & $40,941,246$ \\
11 & 2009 Q3 & $47,782,022$ \\
12 & 2009 Q4 & $49,296,071$ \\
13 & 2010 Q1 & $48,204,412$ \\
14 & 2010 Q2 & $53,435,639$ \\
15 & 2010 Q3 & $54,287,889$ \\
\hline
\end{tabular}

Unit: million NTDs.

\section{Results and Analyses}

There are many factors which could influence the Taiwan economy, such as the country's culture, the government's policy, and the industry framework. Some factors are clear, and others are not clear. But semiconductor still is the major supporting in Taiwan.

IC Industry Situation in Taiwan. Taiwan Semiconductor Manufacturing Company (TSMC) and United Microelectronics Corporation (UMC) are the first and second in worldwide ranking, with a combined market share exceeding $50 \%$. IC assembly industry total product value is the first of the worldwide [13].

Table 1 shows the historical data series of the IC assembly industry Revenue of Taiwan from 2007 Q1 to 2010 Q3, but fluctuating randomly. So this paper forecasts and analyzes IC assembly industry revenue by Grey-Markov forecasting model.

4.1. Build the GM(1,1) Grey Forecasting Model. Using the data of the IC assembly industry revenue of Taiwan from 2007 Q1 to 2010 Q3, in Table 1 and (1)-(7) for model building, the $\mathrm{GM}(1,1)$ model of IC assembly industry revenue yield is

$$
\begin{aligned}
\widehat{Y}(k) & =\widehat{x}^{(0)}(k+1) \\
& =46389938 e^{-0.00887984 k} \quad(k=1,2,3, \ldots, k),
\end{aligned}
$$

where $k$ is the series number of the quarter and $k=1$ means 2007 'Q1.

4.2. Partition of States by Markov-Chain Forecasting Model. According to the actual data, four states, that is, four contiguous intervals, are established about the curve of $X^{(0)}(k+1)$. 


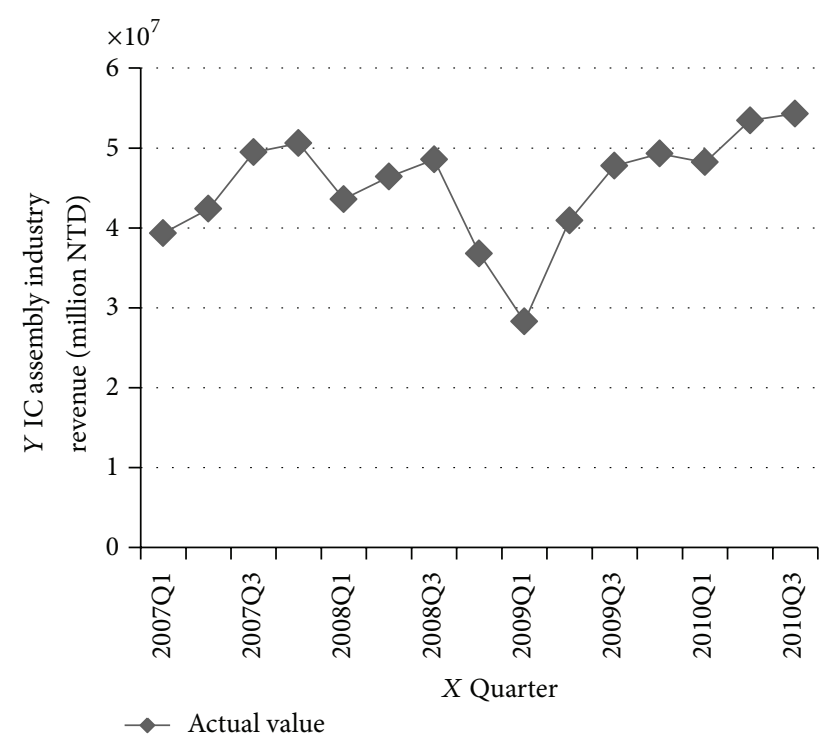

FIgURE 1: The curve of the IC assembly industry revenue in Taiwan.

According to (8)-(9), the four states intervals can be got as follows:

$$
\begin{array}{lc}
E_{1}: E_{11}=\widehat{Y}(k)-1.08 \bar{Y} & E_{21}=\widehat{Y}(k)-0.28 \bar{Y} \\
E_{2}: E_{12}=\widehat{Y}(k)-0.28 \bar{Y} & E_{22}=\widehat{Y}(k) \\
E_{3}: E_{13}=\widehat{Y}(k) \quad E_{23}=\widehat{Y}(k)+0.28 \bar{Y} \\
E_{4}: E_{14}=\widehat{Y}(k)+0.28 \bar{Y} \quad E_{24}=\widehat{Y}(k)+1.08 \bar{Y},
\end{array}
$$

where $\bar{Y}$ denotes the average value of the historical IC assembly industry revenue of Taiwan from 2007 Q1 to 2009 Q4. Figure 1 shows the historical data series and the states intervals.

4.3. Calculation of State Transition. The number of the historical data in every interval can be gotten as follows:

$$
M_{1}=1, \quad M_{2}=3, \quad M_{3}=7, \quad M_{4}=1,
$$

where $M_{i}$ denotes the number of the historical data in the interval $i$ and $i=1,2,3,4$. The numbers of one-step transiting to $E_{1}, E_{2}, E_{3}$, and $E_{4}$ from $E_{3}$ are as follows:

$$
M_{31}=0, \quad M_{32}=1, \quad M_{33}=4, \quad M_{34}=1 .
$$

Next $M_{i j}(1)$, where $i=1,3,4$ and $j=1,2,3,4$, can be calculated in the same way. Then calculate the one-step transition probability to every states interval and present them in the transition matrix $R(1)$ as follows:

$$
R(1)=\left[\begin{array}{cccc}
\frac{0}{1} & \frac{0}{1} & \frac{1}{1} & \frac{0}{1} \\
\frac{1}{3} & \frac{0}{3} & \frac{2}{3} & \frac{0}{3} \\
\frac{0}{7} & \frac{1}{7} & \frac{4}{7} & \frac{1}{7} \\
\frac{0}{1} & \frac{0}{1} & \frac{1}{1} & \frac{0}{1}
\end{array}\right] .
$$

4.4. Calculate the Forecast Value. According to (12), the date of IC assembly industry revenue in 2010 Q1 is calculated; that is,

$$
\begin{aligned}
\widehat{Y}(13) & =\frac{1}{2\left(E_{13}+E_{23}\right)} \\
& =\frac{1}{2(55555326+52066442)}=53810904 .
\end{aligned}
$$

As Table 1 shows, the actual IC assembly industry revenue in Taiwan in 2010 Q1 is 48204412 million NTD. So the precision of the forecasting is 88.37 percent.

By the same method, the IC assembly industry revenue in 2010 Q2 and Q3 can be calculated, the forecast value of 2010 Q2 is $58,636,460$ million NTD, and that of 2010 Q3 is 59105003 million NTD. So the forecasting precisions are $90.27 \%$ and $91.13 \%$.

4.5. Comparison of Forecast Value between the Grey-Markov, $G M(1,1)$, and DGM(1,1) Forecasting Model. First build a $\mathrm{GM}(1,1)$ Grey forecasting model to forecast the IC assembly industry revenue from 2010 Q1 to Q3. And utilize GM(1,1) to build and construct a $5 \mathrm{DGM}(1,1)$ rolling modeling, forecasting in the same way. Then the forecasting results are presented and the results that are forecasted by Grey-Markov model are presented in Table 2.

The forecast value of Grey-Markov forecasting models is more precise than GM(1,1) grey forecasting model in Figure 2 . It is showed that the Grey-Markov forecasting model is better for forecasting the IC assembly industry revenue in Taiwan. Figure 2 is showed that even if it causes the random array to fluctuate too big to face the influence that Financial turmoil, the forecasting curve of $\operatorname{GM}(1,1), \operatorname{DGM}(1,1)$, and Grey Markov, the Grey-Markov forecasting models are still fit for the predication of the IC assembly industry revenue in Taiwan.

\section{Conclusions and Discussions}

Grey Markov prediction model is a hybrid model which mixes traditional grey prediction and Markov-chain, with fewer limitations which not need to complicate matrix operations. By the empirical analysis, we can prove the ability with the advantages of short-term forecasts, and by using $\mathrm{GM}(1,1)$, most future trends can be reflected. Indeed, the Markov probability matrix can extract the information provided by historical data to response to fluctuation system. Even when facing inconsistent fluctuating events, such as the financial crisis, it still gain a high accuracy [12].

In addition to revenue, the impact of IC packaging and testing industry in Taiwan also includes the country economy, industrial structure, policies of government, and even the climate, culture, and other relevant issues. These indicators are followed by specific historical data trend of random fluctuations which are suitable for grey Markov model. It is best to be applied to big fluctuation time-series problems, but outliers often appear in the scope of expectations. According to past experience, it is often impossible for anyone to believe 
TABLE 2: Comparison of forecast values with three different methods.

\begin{tabular}{|c|c|c|c|c|c|}
\hline Year & & Reality amount & $\mathrm{GM}(1,1)$ & $\operatorname{DGM}(1,1)$ & Gray-Markov \\
\hline \multirow{3}{*}{2010 Q1 } & $\mathrm{V}$ & \multirow{3}{*}{$48,204,412$} & 41700971 & 60847214 & 53810904 \\
\hline & $\mathrm{E}$ & & $13.49 \%$ & $26.23 \%$ & $11.63 \%$ \\
\hline & $\mathrm{P}$ & & $86.51 \%$ & $73.77 \%$ & $88.37 \%$ \\
\hline \multirow{3}{*}{2010 Q2 } & $\mathrm{V}$ & \multirow{3}{*}{$53,435,639$} & 41332312 & 67197053 & 58636460 \\
\hline & $\mathrm{E}$ & & $22.65 \%$ & $25.75 \%$ & $9.73 \%$ \\
\hline & $\mathrm{P}$ & & $77.35 \%$ & $74.25 \%$ & $90.27 \%$ \\
\hline \multirow{3}{*}{2010 Q3 } & $\mathrm{V}$ & \multirow{3}{*}{$54,287,889$} & 40966912 & 76155527 & 59105003 \\
\hline & $\mathrm{E}$ & & $24.54 \%$ & $40.28 \%$ & $8.87 \%$ \\
\hline & $\mathrm{P}$ & & $75.46 \%$ & $59.72 \%$ & $91.13 \%$ \\
\hline
\end{tabular}

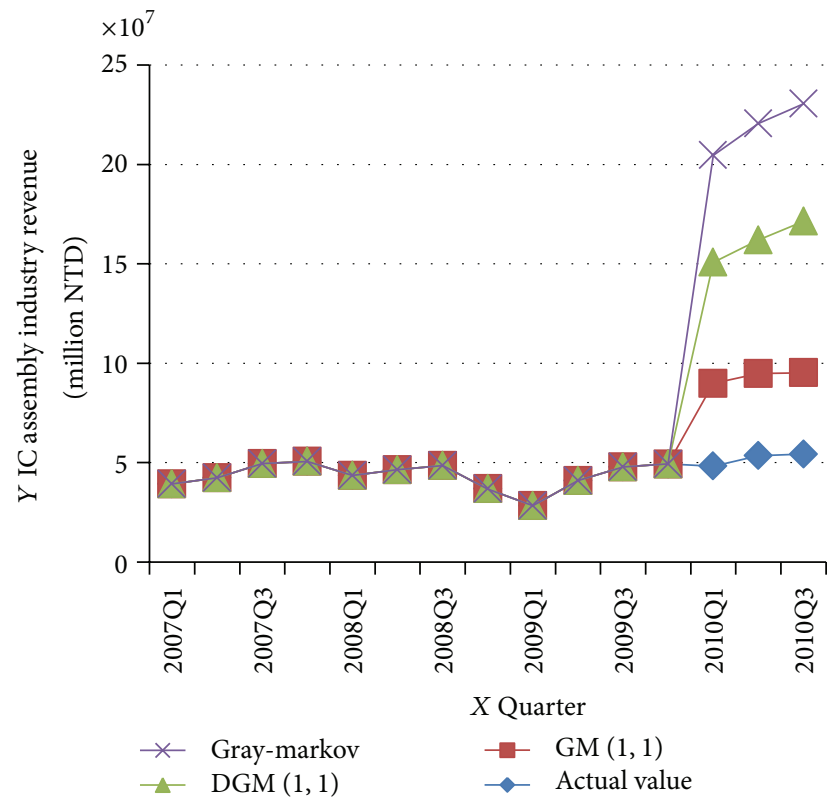

FIgURE 2: The forecasting curve of three different methods.

the possibility of their emergence. With the tremendous impact, we easily fall into the Black Swan myth because of human nature, and let it becomes hard explained and unpredictable.

When enterprise decision-makers make decisions for a major future investment, there must be a risk management mechanism; however, the reference data toward decision making always depend on historical data. For future industry prediction, there are many methods which can be used, but most of them can only reach accurate prediction in normal behavior. In the past market which included drastic noncontrollable factor's changes, instant dropping data could be recognized as special cases or outliers which would not be calculated. But the results may be distorted due to limited or insufficient data. Therefore, this study applied Markov prediction, which includes fewer complicated calculation and higher accuracy, to help the important references and make better investment portfolio decision.

\section{Acknowledgments}

The authors gratefully acknowledge Dr. Chung, Yi Shun from National Cheng Kung University (NCKU) and Mr. Chuang,
Yun Chung from Metal Industries Research \& Development Centre (MIRDC), for their comments on earlier versions of the paper.

\section{References}

[1] N. Nicholas, "The black swan: the impact of the highly improbable," Journal of the Management Training Institut, vol. 36, no. 3, p. 56, 2008.

[2] D. Ju-Long, "Control problems of grey systems," Systems and Control Letters, vol. 1, no. 5, pp. 288-294, 1982.

[3] H. Y. B. Yidan, "Grey-Markov forecasting model and its application," Systems Engineering-Theory \& Practice, vol. 4, p. 12, 1992.

[4] Z. Jun, S. Jianming, and F. Yingjun, “The grey model MGM (1, $n$ ) and its application," Systems Engineering-Theory \& Practice, vol. 5, pp. 109-113, 1997.

[5] Y. He and M. Huang, "A grey-Markov forecasting model for the electric power requirement in China," in Proceedings of the Advances in Artificial Intelligence: 4th Mexican International Conference on Artificial Intelligence (MICAI '05), pp. 574-582, Springer, 2005.

[6] M.-L. Ken and Y.-B. Li, "A study of applying grey predictive module into sales revenue prediction of convenience chain stores an investigation of 7-Eleven and familymart," Journal of Grey System, vol. 12, no. 3, pp. 139-146, 2009.

[7] K.-C. Hung, K.-P. Lin, F.-Y. Hsu, C.-K. Wang, and J.-C. Lin, "Grey model with rolling mechanism for radio-wave path-loss forecasting in suburban environment," Journal of Grey System, vol. 13, no. 2, pp. 49-53, 2010.

[8] G. L. Siqueira, E. A. Vasquez, R. A. Gomes, C. B. Sampaio, and D. A. Borghi, "Comparison between propagation measurements and coverage prediction models for small urban cells," in Proceedings of the Global Telecommunications ConferenceCommunications: The Key to Global Prosperity (GLOBECOM '96), vol. 2, pp. 1182-1186, IEEE, London, UK, November 1996.

[9] C.-T. Lin and S.-Y. Yang, "Forecast of the output value of Taiwan's opto-electronics industry using the Grey forecasting model," Technological Forecasting and Social Change, vol. 70, no. 2, pp. 177-186, 2003.

[10] M.-S. Yin, "Fifteen years of grey system theory research: a historical review and bibliometric analysis," Expert Systems with Applications, vol. 40, no. 7, pp. 2767-2775, 2013.

[11] L. Huang, P. Jin, Y. He et al., "Prediction of silkworm cocoon yield in china based on grey-Markov forecasting model," in Proceedings of the Advances in Artificial Intelligence: 5th Mexican International Conference on Artificial Intelligence (MICAI '06), pp. 505-512, Springer, 2006. 
[12] C.-C. Hsu and C.-Y. Chen, "Applications of improved grey prediction model for power demand forecasting," Energy Conversion and Management, vol. 44, no. 14, pp. 2241-2249, 2003.

[13] C.-C. Lou, T.-P. Lee, S.-C. Gong, and S.-L. Lin, "Effects of technical innovation on market value of the U.S. semiconductor industry," Technological Forecasting and Social Change, vol. 77, no. 8, pp. 1322-1338, 2010. 


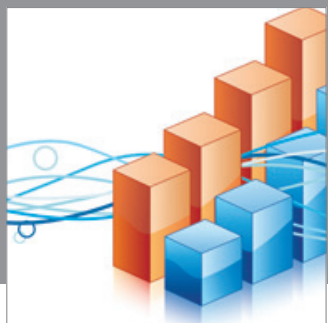

Advances in

Operations Research

mansans

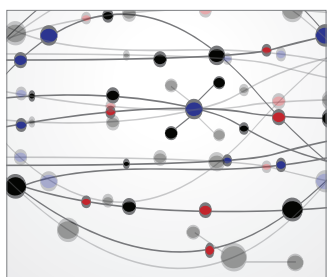

The Scientific World Journal
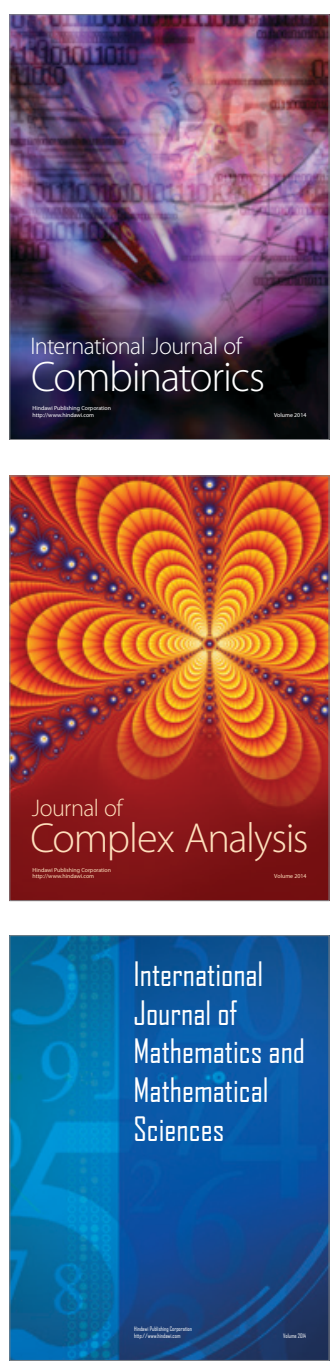
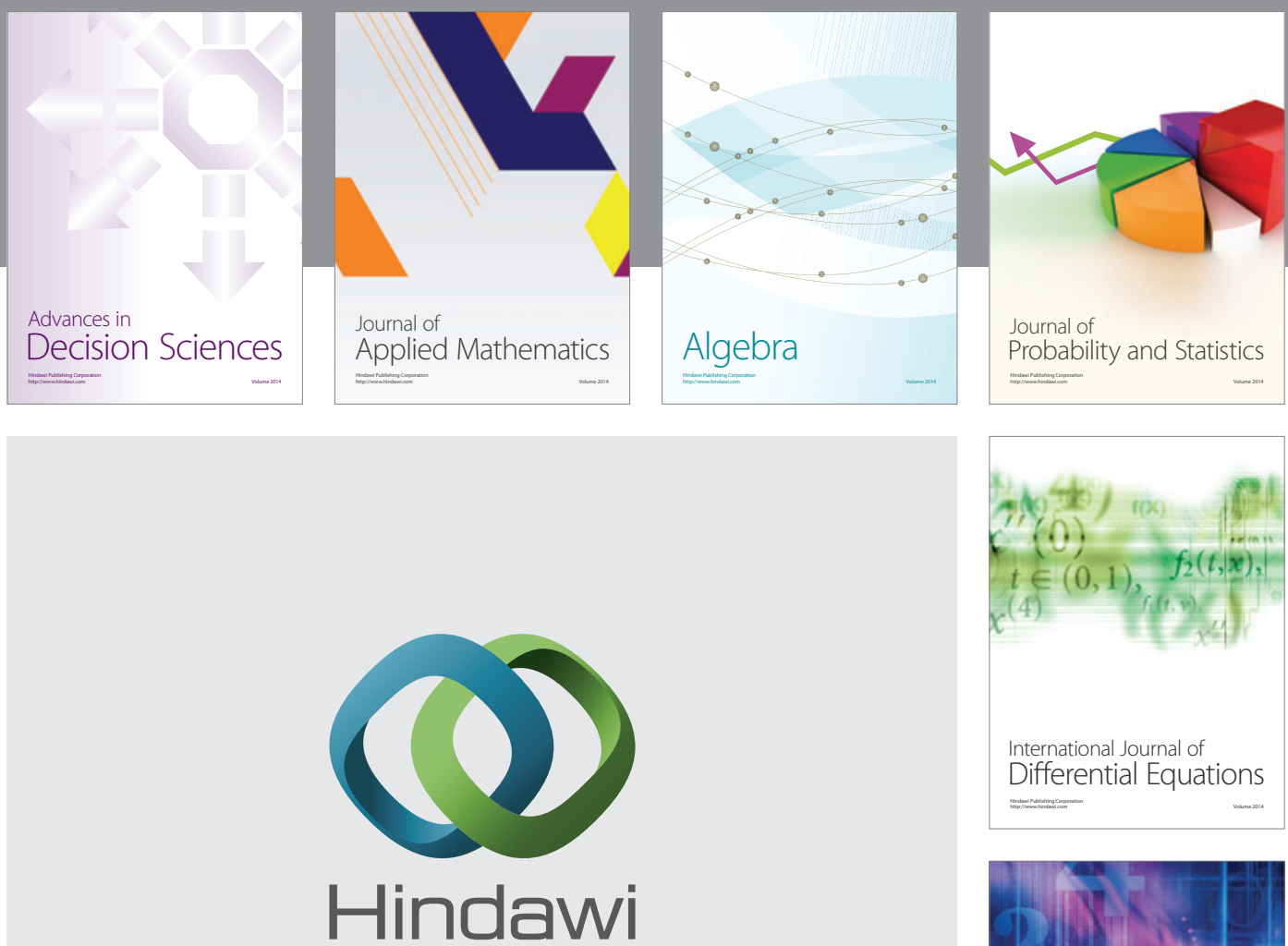

Submit your manuscripts at http://www.hindawi.com
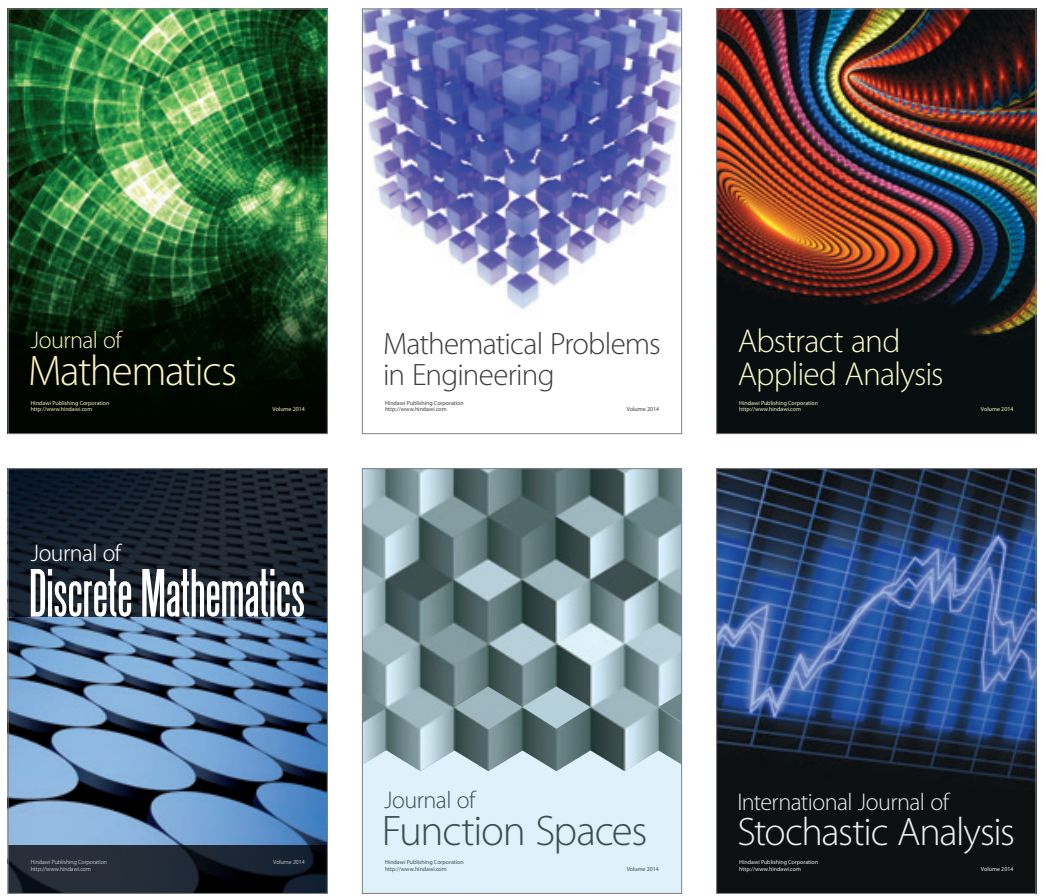

Journal of

Function Spaces

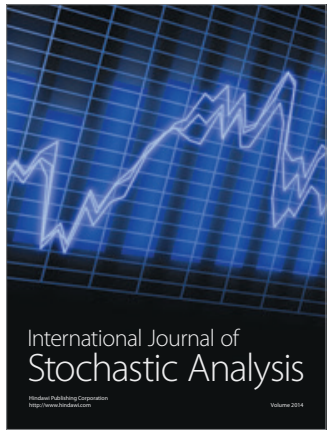

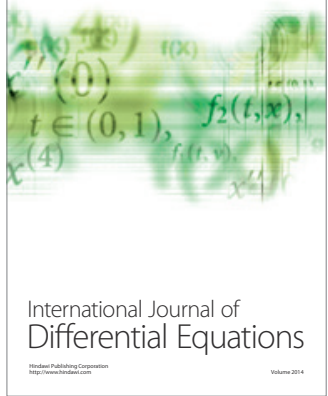
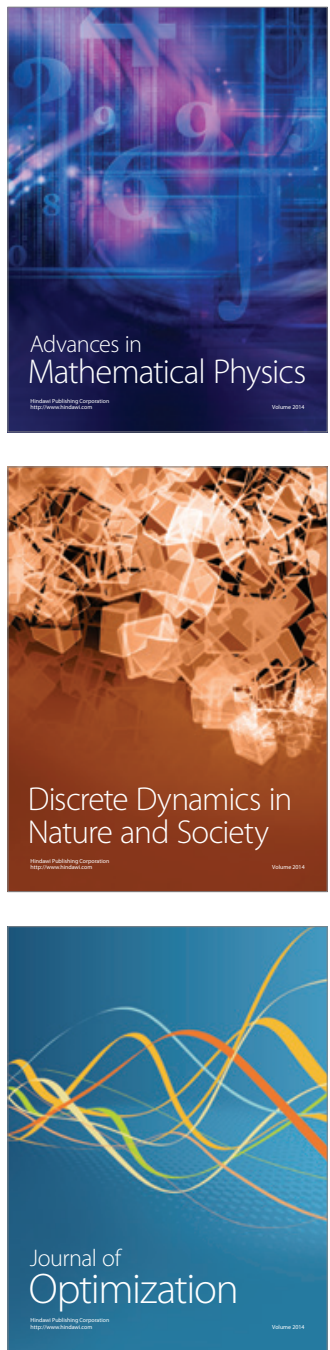